\title{
Animal model investigation suggests betamethasone alters the pharmacokinetics of amikacin
}

\author{
Seth Kwabena Amponsah ${ }^{1}$, Kwabena Frimpong-Manso Opuni ${ }^{2 *}$, Ama Asiedua \\ Donkor ${ }^{1}$ \\ ${ }^{1}$ Department of Pharmacology and Toxicology, School of Pharmacy, University of Ghana, Accra, Ghana \\ ${ }^{2}$ Department of Pharmaceutical Chemistry, School of Pharmacy, University of Ghana, Accra, Ghana
}

*Corresponding Author: E-mail: kfopuni@ug.edu.gh; Telephone: +233208260595

Received: August 30, 2018; Revised: October 25, 2018; Published: November 01, 2018

\begin{abstract}
Corticosteroids, such as betamethasone, are sometimes administered to women who are at risk of pre-term delivery. These corticosteroids cross the placenta to the fetus and decrease respiratory distress syndrome in preterm newborns. Preterm newborns are often susceptible to infections partly due to their immature immune system. Amikacin is one of the aminoglycosides used in the treatment of newborn infections. There is, however, a dearth of information on the effect of prenatal corticosteroids on the disposition of aminoglycosides administered to newborns days later. We evaluated the effect of pre-administration of betamethasone on the disposition of amikacin, $72 \mathrm{~h}$ after last dose of betamethasone, using an animal model. The pharmacokinetic parameters of rats administered betamethasone followed by amikacin vis-avis rats administered saline followed by amikacin were as follows: $C_{\max } 16.6 \mu \mathrm{mol} L^{-1}$ vs. $31.4 \mu \mathrm{mol} L^{-1}$, $A \cup C_{0 \rightarrow 8} ; 26.8 \mu \mathrm{mol} \mathrm{h} L^{-1}$ vs. $153.5 \mu \mathrm{mol} h L^{-1}, K_{e} ; 0.26 \mathrm{~h}^{-1}$ vs. $0.18 \mathrm{~h}^{-1}$, and $t_{1 / 2} ; 2.6 \mathrm{~h}$ vs. $3.9 \mathrm{~h}$, respectively. About a 1.5-fold increase in the elimination of amikacin was observed in the Sprague-Dawley rats pretreated with betamethasone compared with those pre-treated with saline. This ultimately led to differences in the other pharmacokinetic parameters amongst the two groups of rats. Although an animal model investigation showed some level of interaction, a follow-up study in preterm newborns where possible interaction of the two drugs is studied later than Day 1, is recommended.
\end{abstract}

\section{Keywords}

Excretion; Interaction; Preterm

\section{Introduction}

According to the World Health Organization, about 15 million babies (more than 1 in 10 babies worldwide) are born prematurely annually [1]. In 2015, an estimated 1 million preterm deaths were recorded, while many survivors of preterm birth faced life-long morbidities such as learning disabilities and respiratory problems [1]. Newborn care, which includes pharmacotherapy, has been one of the interventions to reduce the burden of morbidity and mortality in this cohort of infants.

Corticosteroids are usually administered to women who are at risk of imminent preterm birth. Betamethasone, a corticosteroid of choice in some resource-poor settings, is known to decrease the occurrence of respiratory distress syndrome in preterm newborns. The main mechanism of betamethasone is to accelerate the development of Types I and II pneumocytes (alveolar cells) of the fetus after the drug 
has crossed the placenta in its active form. Usually, a single course of betamethasone is recommended between 24 and 33 weeks of gestation among pregnant women who are at risk of preterm delivery (often administered within 7 days). Reports suggest that antenatal betamethasone increases glomerular filtration rate (GFR) on Day 3 of the life of preterm newborns [2].

Furthermore, newborns, especially those born prematurely are highly susceptible to infections due to their immature immune system [3]. The cornerstone of treatment of these infections is the use of antibiotics. Amikacin is a commonly prescribed aminoglycoside for empirical treatment of Gram-negative bacterial infections in neonatal intensive care units in most developing countries [4]. Aminoglycosides are known to be excreted almost solely via the kidneys [5]. There is the likelihood, however, that the effect of corticosteroids on GFR may ultimately alter serum levels of aminoglycosides administered to newborns. A decrease in serum levels of aminoglycosides as a result of alteration in GFR by corticosteroids may lead to selection for resistant microorganism strains and infection treatment failure.

A previous study has showed that administration of betamethasone to pregnant mothers had no significant effect on amikacin clearance on the first day of life of preterm newborns [6]. In that study, blood samples for therapeutic drug monitoring were drawn $40 \mathrm{~min}$ before and after completion of the second dose of amikacin [6]. Hence, blood samples for pharmacokinetic analysis were taken between $23-43 \mathrm{~h}$, according to various dosing schemes employed in that study. It is noteworthy, however, that another study concluded that prenatal exposure to betamethasone increased GFR; but this effect was observed on Day 3 $(72 \mathrm{~h})$ of the life of preterm infants [2]. Also considering the fact that amikacin treatment for newborns with infection could range from 5-7 days, there is the likelihood that Day 1 blood samples for pharmacokinetic (clearance) estimations may not reflect the effect of prenatal administration of betamethasone on amikacin. We therefore sought to investigate the effect of pre-administration of betamethasone on the pharmacokinetics of amikacin using an animal model, where blood samples for pharmacokinetic estimates of amikacin were drawn $72 \mathrm{~h}$ after the last dose of betamethasone. An animal model will provide preliminary account of possible pharmacokinetic interaction of these two drugs, and serve as a basis for full clinical studies in humans.

It is noteworthy that the initial experimental design tried to mimic exactly what pertains in humans: administration of betamethasone to pregnant rat, followed by administration of amikacin to litters, and serial drawing of blood from litters for pharmacokinetic estimation. However, this approach had ethical (drawing blood 5 times from rat litter) and operational implications (low blood volumes). Therefore, the current study had to assess possibility of interaction between amikacin and betamethasone using young adult rats.

\section{Experimental}

\section{Experimental Animals}

The study was approved by the Ethical and Protocol Review Committee of the University of Ghana Medical School (Protocol ID: MS-Et/M.8-P.5.3). The animals were handled using procedures and techniques in accordance with the guidelines published by the National Institute of Health for the Care of Laboratory Animals [7].

Twelve (12) healthy male Sprague-Dawley (SD) rats (7 - 8 weeks old, weighing between $200-250 \mathrm{~g}$ ) were obtained and kept under suitable conditions throughout the experimental process. The animal model used only male rats to exclude sex as a confounding factor. A controlled breeding room of temperature 26 
$\pm 2{ }^{\circ} \mathrm{C}$ with a $12 \mathrm{~h} \mathrm{light/dark} \mathrm{cycle} \mathrm{was} \mathrm{used.}$

The SD rats were maintained on local animal feed and water ad libitum. The animals were put into two (2) groups of six (6) - Group 1 and Group 2. The animals in Group 1 (test) were first administered with standard doses of betamethasone and thereafter, standard doses of amikacin. Animals in Group 2 (control) were treated in a similar manner except replacing betamethasone with normal saline.

\section{Drug administration}

A dose of $0.15 \mathrm{mg} \mathrm{kg}^{-1}$ of betamethasone (Diprofos*, Schering-Plough, Brussels, Belgium) was administered intraperitoneally to the test group every $24 \mathrm{~h}$ for 3 consecutive days. Betamethasone dose was based on allometric scaling from humans (prenatal doses of $12 \mathrm{mg}$ given every $24 \mathrm{~h}$ for $2-3$ days) to rats. After this, a dose of $10 \mathrm{mg} \mathrm{kg}^{-1}$ of amikacin (Amikin ${ }^{\circledR}$, Bristol-Myers Pharmaceuticals, Middlesex, England) was administered intraperitoneally to the rats every $24 \mathrm{~h}$ for 3 consecutive days. Of note, similar amikacin dosing in rats has been reported elsewhere [8].

Normal saline was administered intraperitoneally to rats in Group 2, using same volume per weight administration as with betamethasone. The normal saline was administered to SD rats for 3 consecutive days. Afterwards, a dose of $10 \mathrm{mg} \mathrm{kg}^{-1}$ of amikacin was administered via the intraperitoneal route every $24 \mathrm{~h}$ for 3 consecutive days.

\section{Blood sample collection and estimation of serum amikacin level}

Tail vein blood samples were taken following the fourth dose $(72 \mathrm{~h})$ of amikacin after $0.5,1,2,4$ and $8 \mathrm{~h}$, for rats in Groups 1 and 2. Blood samples were collected into microtainer gel tubes and centrifuged at $1,500 \mathrm{rpm}$ for $10 \mathrm{~min}$ to obtain serum.

Samples from SD rats were pooled as a result of low serum volume required for assay. Therefore, one pooled-sample was obtained for each time point for Groups 1 and 2. Usually, challenges associated with low sample volume during pharmacokinetic studies can be circumvented by sample-pooling, which will not alter eventual pharmacokinetic estimation [9]. Fluorescence polarization immunoassay (FPIA), using COBAS INTEGRA $^{\circledast} 400$ (Roche Diagnostic Limited, Basel, Switzerland) as previously described [10] was the assay used in determining serum amikacin levels. The principle behind FPIA is competition for antibody binding sites between drug in sample and drug labeled with an enzyme. The coefficient of variation over the entire calibration range during assay for amikacin was less than $4 \%$.

\section{Pharmacokinetic analysis}

Estimation of pharmacokinetic parameters of amikacin in the two groups was by a non-compartmental analytical approach. The linear trapezoidal rule was used in extrapolating area under the concentrationtime curves (AUCs) for the two groups. AUCs were from time $0 \mathrm{~h}$ till the last measurement point $(8 \mathrm{~h})$. Elimination rate constant $\left(K_{\mathrm{e}}\right)$ was assessed by linear regression of the terminal part of a log of the concentration and time curves. Elimination half-life $\left(t_{1 / 2}\right)$ was calculated as; $t_{1 / 2}=0.693 \mathrm{Ke}_{\mathrm{e}}^{-1}$.

\section{Results and Discussion}

Concentration-time curve of each group after amikacin samples were pooled at the various predetermined times in the SD rats is shown in Figure 1.

Estimates of pharmacokinetic parameters of amikacin in SD rats (Table 1) were obtained from concentration-time curves. Elimination $\left(K_{\mathrm{e}}\right)$ of amikacin was faster in SD rats pre-administered with 
betamethasone compared to SD rats pre-administered with normal saline, and this resulted in a shorter half-life for the former. The maximum amikacin concentration $\left(C_{\max }\right)$ for SD rats pre-administered with normal saline was about two-fold higher than SD rats pre-administered with betamethasone. Total drug exposure from time 0 to $8 \mathrm{~h}\left(\mathrm{AUC}_{0 \rightarrow 8}\right)$ was also about four-fold greater for the SD rats pre-administered with normal saline.

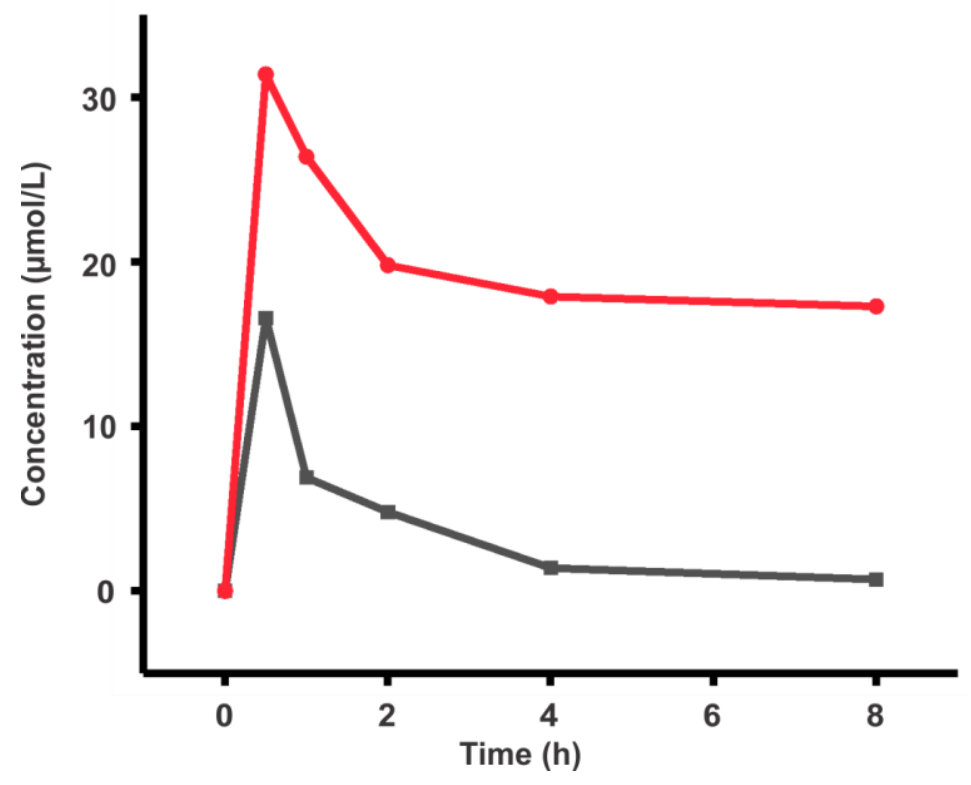

Figure 1. Concentration-time curves of amikacin in rats after pre-treatment with normal saline (red solid line; filled circles) and betamethasone (gray solid line; filled squares)

Table 1. Summary of amikacin pharmacokinetic parameters among two groups of rats

\begin{tabular}{lcc}
\hline PK parameter (Units) & Betamethasone + Amikacin & Normal Saline + Amikacin \\
\hline$C_{\max }\left(\mu \mathrm{mol} \mathrm{L}^{-1}\right)$ & 16.60 & 31.40 \\
$\mathrm{AUC}_{0 \rightarrow 8}\left(\mu \mathrm{mol} \mathrm{L}^{-1} \mathrm{~h}^{-1}\right)$ & 26.80 & 153.50 \\
$K_{\mathrm{e}}\left(\mathrm{h}^{-1}\right)$ & 0.26 & 0.18 \\
$t_{1 / 2}(\mathrm{~h})$ & 2.62 & 3.96
\end{tabular}

Possible causes of resistance to antibiotics include amongst others, drug-drug interactions. Drug interactions leading to low serum levels of antibiotics can result in sub-therapeutic effect or emergence of resistant strains of microorganisms [11]. It is therefore imperative that potential drug interactions involving antibiotics are identified.

In this study, we investigated the effect of pre-treatment of betamethasone on the disposition of amikacin, after $72 \mathrm{~h}$, using an animal model. It is evident that amikacin was eliminated at a faster rate in the betamethasone pre-treated group compared to the normal saline pre-treated group. This led to a shorter half-life $(2.62 \mathrm{~h})$ in the betamethasone pre-treated group compared to the normal saline group (3.96 h). Betamethasone is known to alter kidney function by increasing GFR [2], and this may have consequently led to the increase in renal elimination of amikacin in the betamethasone pre-treated group of this study. The outcome of this work suggests that pre-treatment with betamethasone could increase renal elimination of amikacin, possibly through increasing GFR $72 \mathrm{~h}$ after daily amikacin administration. If indeed this effect observed in the current study occurs in newborns, then there may be a need for dose modification in those who receive prenatal corticosteroids. 


\section{Conclusions}

The current study showed that betamethasone altered the pharmacokinetic parameters of amikacin in Sprague-Dawley rats $72 \mathrm{~h}$ after daily amikacin administration. Although this study was conducted in an animal model, this finding is noteworthy, as this may serve as a basis for future studies in humans where blood samples for pharmacokinetic evaluation of amikacin are taken after Day 3 of the life of preterm newborns.

\section{Acknowledgements}

The authors are grateful to Perseus Nana Asare and Kwabena Antwi, who helped with animal handling. Thanks also go to the technical staff of the Department of Pharmacology and Toxicology, and Department of Pharmaceutical Chemistry, School of Pharmacy, University of Ghana.

\section{References}

[1] Care of the preterm and low-birth-weight newborn. World Health Organization, Geneva, Switzerland.

[2] J.N. van den Anker, W.C. Hop, R. de Groot, B.J. van der Heijden, H.M. Broerse, J. Lindemans, P.J. Sauer. Effects of prenatal exposure to betamethasone and indomethacin on the glomerular filtration rate in the preterm infant. Pediatr Res 36 (1994) 578-581.

[3] J.L. Wynn, J. Neu, L.L. Moldawer, O. Levy. Potential of immunomodulatory agents for prevention and treatment of neonatal sepsis. J Perinatol 29 (2009) 79-88.

[4] A. Siddiqi, D.A. Khan, F.A. Khan, A. Razzaq. Therapeutic drug monitoring of amikacin in preterm and term infants. Singapore Med J 50 (2009) 486-489.

[5] K. Bruce, R.W. Hicks. Perioperative pharmacology: a focus on aminoglycosides. AORN J 93 (2011) 464-468; quiz 469-471.

[6] K. Allegaert, V. Cossey, M. Rayyan, C. Vanhole, H. Devlieger. Administration of betamethasone before birth has no effect on the amikacin pharmacokinetics in preterm infants at birth. Paediatric and Perinatal Drug Therapy 6 (2005) 149-154.

[7] Memorandum of understanding between the Office of Laboratory Animal Welfare Natioanal Institutes of Health U.S. Department of Health and Human Services and the Office of Research Oversight and the Office of Research and Development Veterans Health Administration U.S. Department of Veterans Affairs Concerning Laboratory Animal Welfare. National Institutes of Health, 2007.

[8] H.J. Olguín, M.C. Portugal, J.F. Pérez, A.C. Vieyra, C.F. Pérez, A.A. Rodríguez. Effect of mannitol on the pharmacokinetics of amikacin in wistar rats. Brazilian Archives of Biology and Technology 52 (2009) 835-839.

[9] L.E. Riad, K.K. Chan, R.J. Sawchuk. Determination of the Relative Formation and Elimination Clearance of Two Major Carbamazepine Metabolites in Humans: A Comparison Between Traditional and Pooled Sample Analysis. Pharmaceutical Research 8 (1991) 541-543.

[10] I. Hemmila. Fluoroimmunoassays and immunofluorometric assays. Clin Chem 31 (1985) 359-370.

[11] J. Van Den Anker. Pharmacokinetics and renal function in preterm infants. Acta Paediatrica 85 (1996) 1393-1399. 